\title{
Devenir sujeto. Una aproximación sociológica
}

\author{
Becoming a subject. A sociological approach \\ MarVenegas / mariter@ugr.es \\ Universidad de Granada, España
}

\begin{abstract}
An overview of some milestones in sociological theory leads to the review of some key contributions of interest to the sociological discussion on the process of becoming a subject. This paper presents the results of such overview, guided by the two major micro-sociological processes involved in the formation of the subject, socialization and subjectivation, and tries to formulate a single theory that emphasises the complementarity of both processes in the formation of the subject within the frame of social structure and its macro-sociological processes of social reproduction and change. In doing so, the starting point is the thesis that a subject is produced between constriction and possibility. The overview is concretized in the field of Education. The paper concludes with the formulation a theory in which action, subject and structure are interconnected through processes of both maintaining and challenging social structures, in so far as the reflexivity of practice makes social change possible.
\end{abstract}

Key words: socialization, subjectivation, subject, action, structure.

Resumen: Un recorrido por algunos hitos en la teoría sociológica permite revisar algunas contribuciones de interés para una discusión sociológica sobre el proceso de devenir sujeto. Este artículo recoge los resultados de ese recorrido, guiado por los dos grandes procesos microsociológicos implicados en ello: socialización y subjetivación; y trata de formularlos en una propuesta única, sintetizadora y holística, que enfatiza la complementariedad de ambos procesos enmarcados en la estructura de la sociedad y sus procesos macrosociológicos de reproducción y cambios social. Para ello, parte de la tesis de que el sujeto se produce entre la constricción y la posibilidad. El recorrido se concreta en el ámbito de la educación. El artículo concluye formulando una teoría donde acción, sujeto y estructura quedan interconectadas en procesos de mantenimiento de las estructuras sociales, así como de desafío a las mismas, al introducir, mediante la reflexividad de la práctica, la posibilidad del cambio social.

Palabras clave: socialización, subjetivación, sujeto, acción, estructura. 


\section{Introducción}

El proceso de devenir sujeto constituye un objeto de estudio recurrente para las diversas disciplinas que integran las ciencias sociales.

En ese espacio interdisciplinar de pensamiento, este artículo presenta un recorrido teórico por los dos grandes procesos implicados en el devenir sujeto: la socialización y la subjetivación, circunscribiendo el análisis al ámbito de conocimiento de la sociología, ${ }^{1}$ a fin de formular una propuesta teórica que recoja las aportaciones más destacadas cronológicamente para la sociología sobre el devenir sujeto desde una teoría de la acción, que incorpora contribuciones postestructuralistas de especial relevancia para entender este fenómeno, con el objetivo de superar las limitaciones de esas contribuciones relevantes previas, para formular una propuesta teórica sintetizadora y holística.

La siguiente cita de Alain Touraine (2005: 129) establece los márgenes del análisis que se ha querido plantear en este artículo:

El sujeto se forma en la voluntad de escapar a las fuerzas, reglas y poderes que nos impiden ser nosotros mismos, que tratan de reducirnos al estado de un sistema y de su control sobre la actividad, las intenciones y las interacciones de todos. Esas luchas contra lo que nos arrebata el sentido de nuestra existencia son siempre luchas desiguales contra un poder, contra un orden. No hay sujeto si no es rebelde, divido entre la cólera y la esperanza.

De esta cita, se concluye que el sujeto "se forma” entre la constricción y la posibilidad. Aunque cada corriente teórica aborda este hecho de una manera epistemológicamente diferente (como se analizará en este artículo), ha sido constante la producción de trabajos que nos permiten pensar en este fenómeno sociológico a partir de esa disyuntiva que es la formación del sujeto simultáneamente entre constricción y posibilidad.

Los trabajos que se discuten en este artículo no agotan la ingente producción de conocimiento sobre este tema en ciencias sociales, ${ }^{2}$ pero sí son representativos de las contribuciones para el debate sociológico materializado en el ámbito educativo, esto es, para la sociología de la educación. Así, la primera parte del artículo presenta el recorrido por los trabajos revisados para, en la segunda parte, formular la propuesta teórica más holística con la que este texto pretende contribuir al análisis del devenir sujeto.

1 Sin duda, todas las disciplinas que integran las ciencias sociales tienen importantes consideraciones que aportar a este debate; sin embargo, quedan fuera del alcance de este artículo.

2 Algunos ejemplos destacados son David (2006), Quintana y Manrique (2012) y Cervulle (2012). 


\section{Primeras contribuciones: educación, socialización y subjetivación (parte I)}

La educación ha sido el ámbito de desarrollo y aplicación de los conceptos de socialización y subjetivación por excelencia (Durkheim, 1975; Dubet y Martuccelli, 1996 y 1998b; David et al., 2006; Davies, 2006; Venegas, 2012 y 2013a; Youdell, 2006a, 2006b y 2010; Weiss, 2012).

De ahí que el primer referente considerado aquí sea Èmile Durkheim (1975), por ser pionero en definir la educación como fenómeno social, diferenciando entre sus dimensiones espiritual y material, ${ }^{3}$ y contextualizándolo sociohistóricamente: cada sociedad tiene sus propias prácticas e instituciones, e impone sobre sus miembros un sistema educacional con un entramado normativo que provoca constreñimiento estructural, ${ }^{4}$ fruto de la vida en común.

Según este autor, los cambios en el sistema son posibles, pero sólo desde adentro. La educación es, para él, el medio que prepara en el espíritu de niños y niñas las condiciones de su propia existencia, una acción que ejerce la generación adulta sobre la joven. ${ }^{5}$ Sin embargo, la visión funcionalista de Durkheim establece los límites de su análisis: sólo repara en la transmisión de la sociedad al individuo. Faltan aún muchos elementos para completar la imagen que busca esbozar este artículo.

En referencia a esa visión funcionalista iniciada por Durkheim, Robert William Connell (1987: 30) afirma que hacia 1930 se había consolidado la idea de rol social: "Un guión socialmente provisto para el comportamiento individual, primero aprendido y después representado", ${ }^{6}$ siendo la socialización el proceso que posibilita este aprendizaje, mediante el cual los individuos se insertan en las relaciones sociales de las diferentes agencias de socialización.

3 Dimensiones ambas centrales también en la posterior descripción de la construcción social de la realidad de Berger y Luckmann (2008).

4 Ya en Durkheim aparece la idea de constreñimiento estructural como fuerza central en la formación del sujeto, tema clave hasta la actualidad.

5 Esta concepción se encuentra próxima a la posterior noción de socialización de Berger y Luckmann (2008).

6 La traducción de las citas originales en inglés al español fueron realizadas por la autora de este artículo. 
Connell (1987: 50) señala dos razones para desechar esta teoría: 1) elude el análisis de las relaciones de poder, que son las que permiten explicar la resistencia al mismo; sin ellas, el resultado es un modelo de sociedad estático, sin posibilidad de cambio social, ya que ignora el conflicto; 2) adolece de voluntarismo: "Introduce el problema donde empieza lógicamente la teoría social, la relación entre agencia personal y estructura social; pero lo evade disolviendo la estructura en la agencia”.

Para superar las debilidades de la teoría funcionalista de la socialización, Connell (1987 y 2002) afirma que la subjetividad se construye a partir de la práctica del sujeto, desde un enfoque relacional y atendiendo a las bases estructurales de la práctica. Esta tesis ocupa un lugar central en la propuesta teórica de este artículo, junto a la tesis feminista de que todas las relaciones sociales están mediadas por el poder, principio generador de conflicto y resistencia que abre espacio teórico para el análisis de posibles procesos de cambio social (Bartky, 1988; Bordo, 1988 y 1995; Butler, 1997 y 2001; Esteban, 2001; Foucault, 1989; Gil, 2013; Maquieira, 2001; Narotzky, 1995; Ortner, 1993; Venegas, 2013a). ${ }^{7}$

En la década de 1960, Peter Berger y Thomas Luckmann (2008), al describir la construcción social de la realidad, retoman la idea durkheimiana de que la realidad social existe tanto objetivamente (a nivel material) como subjetivamente (a nivel espiritual o de la conciencia humana). Son pioneros en dar una definición constructivista de la socialización primaria como mecanismo central que posibilita esa construcción social. Individuo y sociedad se construyen mutuamente en un proceso dialéctico. El individuo no nace como ser social, sino con la predisposición a serlo, y es la socialización lo que posibilita esa pertenencia.

El punto de partida es la internalización, mediante la cual el individuo asume el mundo (objetivo) que ya existe y es subjetivamente significativo para sus semejantes: lo interpreta y lo hace suyo, siendo "los adultos quienes disponen las reglas del juego" (Berger y Luckmann, 2008: 168-169), concepción similar a la de Durkheim; y añaden que "el individuo llega a ser lo que los otros significantes lo consideran” (Berger y Luckmann, 2008: 165), mediante una dialéctica entre la identidad objetivamente atribuida y la que se asume subjetivamente. Esta dialéctica apunta ya a la subjetivación, si bien ellos no la consideran aún en este proceso.

7 Se citan aquí algunos referentes que muestran abiertamente esta tesis, común a prácticamente la totalidad de trabajos feministas revisados. 
Serán François Dubet y Danilo Martuccelli (1996, 1998a, 1998b) quienes reparen, por primera vez, en la relación entre educación, socialización y subjetivación, desde su sociología de la experiencia. Sin ignorar las funciones de reproducción de la escuela, se centran también en las de producción. Para ellos, coexisten dos términos: la socialización, referida a la formación de actores sociales a través de la interiorización de normas y modelos; y la subjetivación, que se refiere a la formación de sujetos autónomos mediante el distanciamiento con su socialización. Como Connell, Dubet y Martuccelli consideran que actores y estructuras sociales se construyen recíprocamente, por lo tanto reconocen una correlación entre posiciones sociales y dimensiones subjetivas (Bourdieu 2005; Skeggs 1997) que, sin embargo, Connell no comparte en su totalidad.

Haciendo una síntesis de la literatura revisada en este apartado, se constatan dos tesis nucleares para la propuesta teórica que se pretende formular en este trabajo:

Tesis 1: En el nivel de la práctica (microsociológico) es posible hallar procesos de una doble naturaleza que dibujan el primer continuum central:

- La internalización de valores y normas predominantes en una sociedad, consecuencia de la socialización (Berger y Luckamm, 2008; Dubet y Martuccelli, 1996, 1998a, 1998b; Durkheim, 1975; Komarovski, 1946, 1950 y 1954 citado en Connell, 1987; Parsons, 1942 citado en Connell, 1987).

- La resistencia a esos valores y normas, fruto de la subjetivación (Dubet y Martuccelli, 1998b).

Tesis 2: A nivel de la estructura social (macrosociológico), es posible hallar procesos de una doble naturaleza que dibujan el segundo continuum central: la reproducción y el cambio sociales (Connell, 1987; Dubet y Martuccelli, 1998b; Ortner, 1993).

Se ponen así en juego, hasta aquí, los tres paradigmas teóricos clásicos en sociología, en función de su objeto de estudio: actor social y su subjetividad; acción social (lógicas de acción); y estructuras sociales. Sin embargo, las corrientes críticas de la década de 1970 y la tendencia, desde la de 1980, a integrar los niveles de análisis micro (actor, acción) y macrosociales (estructuras) en una misma teoría (Adkins, 2002; Connell, 1987, 2002; Narotzky, 1995; Ortner, 1993) van a abrir espacios a los que se sumará, desde la década de 1990, el cuestionamiento de la lógica binaria y el énfasis tanto en la dimensión discursiva como en las posibilidades de cambio social, todo lo cual hace germinar con fuerza la idea de subjetivación, como se muestra en los dos apartados siguientes. 


\section{De la socialización a la resistencia, la agencia y la práctica}

Desde la década de 1980, la teoría social feminista introduce elementos teóricos que marcan el paso hacia un enfoque teórico más discursivo y menos materialista, más cultural y menos social (Adkins, 2002; Barret, 1992; Touraine, 2005). Además, se defiende con fuerza la tesis de que la práctica es el elemento ontológico que imbrica los niveles micro y macrosociológicos (Connell, 1987, 2002; Nartozky, 1995; Ortner, 1993).

Si acción (o práctica) e interacción cotidianas, junto a las relaciones sociales (fruto y posibilitadoras de las mismas), están en la base de la construcción social de las estructuras sociales que, una vez cristalizadas en la forma de instituciones, aparecen como externas y coercitivas (parafraseando a Durkheim), entonces a la sociología le corresponde dar cuenta de esos procesos, de la conexión entre los mismos, para explicar cómo acción y estructuras se conectan en los procesos sociales de reproducción y cambio. Esta tarea se aborda a continuación.

La práctica puede describir dos procesos microsociológicos diferentes (Ortner, 1993): socialización o resistencia. Siguiendo esta propuesta de Ortner, podemos clasificar las teorías revisadas según un esquema que estructura la práctica como resultante de:

La socialización, de dos tipos:

a) Carente de conflicto social, como plantea el funcionalismo estructural, según se explicó con anterioridad.

b) O considerando el conflicto social, como hacen las teorías estructuralistas de la reproducción social y cultural, según explican Connell (1987, 2002) o Holland y Eisenhart (1990).

La resistencia a las imposiciones estructurales - caso de las teorías de la producción cultural o de la resistencia- que, sin embargo, conduce igualmente a la reproducción estructural (Holland y Eisenhart, 1990; McRobbie, 1987; Willis, 1988 y 2003).

Si la socialización es la posibilitadora de la reproducción social, y la resistencia como producción cultural termina reproduciendo las estructuras sociales igualmente, debe haber algún otro mecanismo que nos permita explicar los procesos históricos de cambio social (como ha ocurrido en el ámbito del género y la sexualidad, por ejemplo).

La teoría social crítica muestra dos corrientes interpretativas en torno al concepto de resistencia: 
- Una interpretación materialista de la producción cultural, entendida como resistencia, ${ }^{8}$ que toma la práctica como unidad de análisis (Bourdieu, 2005; Connell, 1987 y 2002; Holland y Eisenhart, 1990; McRobbie, 1978; Skeggs, 1997; Willis, 1988 y 2003). Así definida, la práctica contiene un potencial de cambio social (Connell, 1987 y 2002), si bien, desde este enfoque, la práctica termina afirmando la reproducción social, pues adolece de varias debilidades (Holland y Eisenhart, 1990), se centra en las relaciones entre el grupo privilegiado y el que no lo es, olvidando las tensiones dentro de cada categoría, la política interna a cada grupo y la formación de las subjetividades, en lo cual residen las claves para definir la práctica como motor de cambio social, como sí lo hace la teoría de la práctica.

- Una interpretación discursiva del poder, que permite, partiendo del concepto de microfísica del poder de Foucault (1989), entender los procesos sociales como prácticas discursivas de sumisión [constricción], al tiempo que entrañan resistencia [posibilidad] (Bartky, 1988; Bordo, 1988 y 1995; Butler, 1997; Weeks, 2003; Youdell, 2006a, 2006b y 2010), introduciendo un concepto postestructuralista de subjetivación que nos permite pensar en la resistencia para el cambio social. Esta resistencia se entiende, pues, como contestación. El poder discursivo del que habla Foucault no es el poder organizado, institucional, burocrático (Connell, 2002), ni el Derecho o las relaciones de dominación/subordinación (Bourdieu, 2005); sino que implica una multiplicidad de relaciones de fuerza, el juego que las transforma, los apoyos y las contradicciones presentes en esas relaciones. Poder como imposición, y resistencia como respuesta a esa imposición son dos dimensiones del mismo proceso, de ahí que el poder haya de ser entendido como generador de resistencias y no desde una lógica binaria de oposición dialéctica, como ocurre en la visión marxista del conflicto social que siguen las teorías de la reproducción y las de la resistencia. Así, señala Foucault (1989) que el poder está en todas partes, de manera que allí donde hay poder, hay resistencia al mismo (Bartky, 1988; Bordo, 1988; Weeks, 2003), de lo que deriva la idea de agencia discursiva (Butler, 1993; Davies, 2006; Youdell, 2006a).

8 Se trata de las teorías de la producción cultural o resistencia, que se originaron en la Escuela de Birminghan en la década de 1970 y cuyos análisis se centraban principalmente en colectivos sociales desfavorecidos, como las clases obreras o las minorías étnicas.

9 Original de 1986. 
De este modo, resistencia, agencia y cambio social aparecen estrechamente vinculados a través de la práctica.

Para superar las debilidades teóricas de las teorías de la producción y de la resistencia, autores como Connell (1987 y 2002), Holland y Eisenhart (1990) u Ortner (1993) optan por la Teoría de la práctica, que emerge en la década de 1980, con el interés por estudiar acción y estructura como dos dimensiones complementarias para entender la realidad social y explicar los procesos de producción, reproducción y cambio del sistema desde la práctica, la cual se define como toda forma de acción humana concebida en términos políticos. Ortner (1993) recoge el presupuesto fundamental de la Teoría de la práctica en los términos de la construcción social de la realidad de Berger y Luckmann (2008): la sociedad es un sistema, el sistema es poderosamente constrictivo, pero puede ser hecho y deshecho a través de acción e interacción humanas (Ortner, 1993).

Es posible diferenciar dos grandes corrientes entre las teorías de la práctica herederas del estructuralismo:

- De la práctica a la estructura, dando primacía al cambio. Connell (1987 y 2002) reconoce la reproducción, pero sólo como aparente, pues afirma que lo que sucede es un proceso constante de cambio derivado de la práctica humana y las "tendencias de crisis". Enfatiza la agencia como sujeto de prácticas que conllevan el cambio desde la perspectiva historicista. La "(e)structura social expresa los constreñimientos que subyacen a una forma dada de organización social” (Connell, 1987: 92), pero “(e)star presente en la práctica cotidiana, hace a la estructura vulnerable de cambios mayores de la práctica” (Connell, 1987: 93). Holland y Eisenhart (1990) hacen suya la mayoría de supuestos teóricos de Connell al desarrollar su Teoría de la práctica.

- De la estructura a la práctica, dando prioridad a la reproducción. Bourdieu (2005) se muestra más próximo a la idea de inmanencia de las estructuras sociales, que toma forma en la noción de habitus, en tanto que las estructuras cognitivas, configuradas a partir de la organización material del campo, orientan la práctica. El autor no niega totalmente las posibilidades de cambio social, si bien afirma que la salida sólo es posible mediante "una transformación radical de las condiciones sociales de producción” (Bourdieu, 2005: 58) de ese habitus. 
Por su parte, las décadas de 1990 y 2000 van a dar paso a un eclecticismo teórico que tiene algunos rasgos destacados como el énfasis en la fragmentación, la diversidad y la deconstrucción (McRobbie, 1994). A principios de los noventa, la posmodernidad va a cuestionar algunos pilares de la modernidad occidental tales como la identidad como esencia, la historia como lineal y evolutiva, el etnocentrismo, un conocimiento clasista, o el biologicismo sobre el que se asienta la lógica binaria (Nicholson, 1990). Barret (1992) retoma el debate foucaultniano entre "las palabras y las cosas"10; esto es, entre lo simbólico y lo material, lo subjetivo y lo objetivo, lo cultural y lo social, y afirma el viraje desde 1980 de las disciplinas sociales hacia las culturales (Adkins, 2002; Touraine, 2005).

Este viraje implica un giro teórico central de lo material (economía, trabajo) a la agencia y la reflexividad. A ello se suma el cuestionamiento de las lógicas binarias, el énfasis en la fluidez, la contingencia, la subjetividad o la centralidad de los discursos. Esta transición teórica es la que nutre de contenido a la corriente postestructuralista. Adkins (2002) destaca el paso de la escala macrosociológica de análisis, más propia de la modernidad, a la escala más micro de la vida cotidiana, la destradicionalización y la individualización (Beck y Beck-Gernsheim, 2001). Adkins (2003: 22) sitúa la reflexividad en el centro de la sociología contemporánea y la define como "reflexión crítica sobre convenciones sociales, normas y expectativas prevalecientes". El valor sociológico de la reflexividad radica en su relación con el cambio social, tal como lo plantea Bourdieu (2005) al señalar que la "toma de conciencia" genera espacios para el cambio social. Aquí radica, precisamente, el valor del concepto de reflexividad en relación con el proceso de subjetivación y el consecuente potencial de cambio social.

También en este marco postestructuralista, la práctica es elemento ontológico de análisis, estrechamente vinculado con resistencia y agencia. Un ejemplo es la obra de Jeffrey Weeks (2003), quien adopta una perspectiva conflictual y constructivista, donde la agencia es un concepto ligado al fenómeno social de la resistencia. Frente a las propuestas funcionalistas que enfatizan la aceptación y reproducción de roles, Weeks enfatiza el papel de la subjetividad y la agencia humanas.

Por su parte, desde su feminismo corpóreo, Elizabeth Grosz (1987) sitúa la agencia en el cuerpo como espacio de resistencia y cambio. Barret (1992) señala la necesidad de elaborar una idea de agencia que supere las debilidades tanto del postestructuralismo antihumanista, como del modernismo humanista.

10 Parafraseando la obra de Michel Foucault "Las palabras y las cosas" que fue publicada originalmente en 1966 y es citada por Michèle Barret (1992). 
Butler (1993: 15) define "la agencia como una práctica reiterativa o rearticulatoria, inmanente al poder en lugar de una relación externa de oposición al poder", por lo que habla de "agencia discursiva" (Youdell, 2006a). En tanto, Allen (2005) enfatiza la necesidad de una teoría del sujeto erigida sobre su subjetividad.

De todo ello emerge un concepto postestructuralista de subjetivación más sistematizado y complejo que el propuesto por Dubet y Martuccelli (1996, 1998a y 1998b), y que es analizado en el siguiente apartado.

\section{La centralidad del sujeto y la subjetivación (parte II)}

El primer referente de relevancia en este sentido lo constituye la obra de Judith Butler (1997), que analiza "las teorías sobre la sujeción", donde desarrolla interesantes ideas planteadas antes en el pensamiento de Foucault. Para Butler, el poder tiene una forma psíquica que define la identidad del sujeto. Butler describe la formación del sujeto a partir de ese doble proceso de sumisión/resistencia, siguiendo el modelo discursivo del poder de Foucault (1989), que la lleva a considerar la forma psíquica del poder como discurso que fundamenta la sujeción, la cual posibilita tanto la subordinación, a lo que se refiere como "estar sujeto", como la formación del sujeto, el "ser sujeto": la subordinación establece las condiciones de posibilidad del sujeto, pues el poder genera resistencia a ese poder. ${ }^{11}$

Butler sigue el pensamiento de Nietzsche y Freud para referirse a la formación de la conciencia como efecto de una prohibición internalizada, de lo que emerge la reflexividad, pues la conciencia es el medio por el cual el sujeto se convierte en objeto para sí mismo. Vemos, de nuevo, esa doble dimensionalidad, al referirse a un sujeto que reflexiona (hacia dentro) y refleja (hacia fuera).

Así, Butler (1997) define la subjetivación como devenir del sujeto, tanto por estar sujeto (sujeción), como por ser sujeto. Considera la subjetivación como un proceso, fruto de la producción discursiva de las identidades. Y añade que las posibilidades de resistencia a la regularización discursiva residen en la psique, en el inconsciente. La resistencia es, pues, efecto del poder, la autosubversión del poder mismo. Siguiendo a Foucault, localiza esa resistencia siempre en el seno de las relaciones sociales, que son ineludiblemente relaciones de poder, como ya se señaló con anterioridad en este artículo.

11 Esta simultaneidad - estar y ser sujeto- constituye el punto de partida de la propuesta que se quiere formular en este artículo, según la cual el poder es externo al sujeto (constriñendo la acción), al tiempo que es la propia jurisdicción del sujeto (posibilitando la acción). 
Pero su planteamiento se relega al nivel de los discursos y al efecto de éstos sobre la psique del sujeto; por lo tanto, tiene la debilidad de necesitar una concreción más sociológica, que sí encontramos en algunos trabajos en sociología de la educación como, en primer lugar, el de Deborah Youdell (2006a), quien analiza las contribuciones de las teorías postestructuralistas sobre poder y sujeto a la comprensión de las desigualdades educativas en la lucha por la justicia social, en lo que sigue a Butler. Su objetivo es mostrar por qué persisten la desigualdad y la discriminación. Para ello, alude a la constitución performativa del sujeto, afirmando que el mero acto de designar constituye al sujeto, o sea, que el sujeto no puede darse por supuesto como preexistente. Para funcionar, la performatividad tiene que producir sentido de aquello que nombra. Emerge así un sujeto político, implicado en formas políticas (politics) y de resistencia (Youdell, 2010) que pueden abrir espacios de acción y cambio social. La subjetivación implica, pues, sujeción al poder y reconocimiento como sujeto.

En segundo lugar, encontramos el trabajo de Bronwyn Davies (2006), similar al de Youdell. Toma al proceso de subjetivación (o subjetivización o subjeción) como proceso por el cual uno se convierte en sujeto, siguiendo la afirmación de Butler de que control y sumisión ocurren simultáneamente en el devenir sujeto. Davies señala que, en el ámbito educativo, estudiantes y profesorado suelen concebirse en términos del humanismo liberal, como individuos autónomos con cierto margen de libertad para elegir a la persona que quieren ser; sin embargo, Butler opta por la subjetivación para enfatizar el proceso a través del cual llegamos a ser quienes, y lo que, somos: "El control y la sumisión son la condición de posibilidad para el sujeto mismo" (Butler citada en Davies, 2006: 426).

La formación del sujeto depende de poderes externos a él. El sujeto puede tanto resistir como agonizar, pero no pre-existe a estos procesos. Las condiciones de posibilidad del sujeto radican en el discurso, así como en los actos sociales mutuamente constitutivos. Para Davies, este proceso de sumisión y control en la formación del sujeto muestra los actos mutuos de reconocimiento a través de los cuales los sujetos se conceden el estatus de sujeto.

Como vemos, estas aportaciones recientes se centran más en la subjetivación al analizar el devenir sujeto. Enfatizan la posibilidad partiendo de la constricción, pero dejan a un lado las contribuciones previas relativas a la socialización. La necesidad de formular una propuesta teórica que vincule ambos procesos de manera complementaria -buscando dar cuenta del devenir sujeto a partir de ellas- motivó la razón de ser de este trabajo. Lo que sigue en este artículo es un intento por ofrecer una propuesta holística y de síntesis 
en torno al devenir sujeto -en inglés, becoming a subject-, que contiene la idea de proceso como algo abierto, inacabado, que se mueve entre continuos: esa búsqueda del sujeto por hacerse, por definirse, como un devenir más que como un producto.

\section{Discusión y propuesta teórica: devenir sujeto desde una teoría postestructuralista de la acción}

La primera definición social de educación (Durkheim, 1975) sienta las bases de lo que será la socialización primaria desde un enfoque constructivista (Berger y Luckmann, 2008), entendida como internalización del mundo social en la conciencia del individuo. Dubet y Martuccelli (1996, 1998b) son los primeros en señalar la complementariedad entre "la socialización, la formación de actores sociales, y la subjetivación, la formación de sujetos autónomos" (Dubet y Martuccelli, 1998b: 12), afirman la existencia de un conflicto entre estos dos procesos del devenir sujeto, del que entienden emerge la resistencia.

En la década de 1980 aparece una nueva orientación teórica (Ortner, 1993): la teoría de la acción o de la práctica (Bourdieu, 2005; Connell, 1987; Giddens, 2004; Holland y Eisenhart, 1990), que permite estudiar acción y estructura sociales como dos elementos complementarios en la comprensión del mundo social, con el objetivo de explicar los procesos de producción, reproducción y cambio del sistema a partir de la acción social. El sistema da forma a la práctica, y, a su vez, la práctica da forma al sistema, de lo cual resultan dos tipos de efectos: la reproducción del sistema de normas, valores y esquemas conceptuales y estructuras sociales, a través del proceso de socialización; o el cambio del sistema, como resultado de relaciones sociales asimétricas o posiciones sociales diferentes.

Desde la década de 1990 encontramos una serie de aportaciones postestructuralistas relativas a resistencia, agencia y cambio, que abren una idea de subjetivación, desde la definición feminista de que toda relación social está mediada por el poder (Bartky, 1988; Bordo, 1988 y 1995; Butler, 1997 y 2001; Connell, 1987 y 2002; Esteban, 2001; Foucault, 1989; Gil, 2013; Maquieira, 2001; Narotzky, 1995; Ortner, 1993; Venegas, 2013a). Así, el análisis del devenir sujeto entre la socialización y la subjetivación se plantea a la luz del debate postestructuralista sobre constricción y posibilidad simultáneamente (Butler, 1997; Davies, 2006; Foucault, 1989; Weeks, 2003; Youdell, 2006a). 
Es decir, la socialización no remite sólo a la constricción y la subjetivación a la posibilidad (como señalan Dubet y Martuccelli (1998b)), sino que la subjetivación - pensada desde las teorías postestructuralistas- nos permite observar la constricción (fruto de la socialización) y la posibilidad (fruto de la subjetivación de la que hablan esos dos autores) como simultáneas en la construcción cotidiana del sujeto a través de su práctica (discursiva y material). Ésta es la tesis que defiende este artículo y que se argumenta a continuación.

La propuesta que se defiende aquí busca formular una teoría del devenir sujeto que incorpora socialización y subjetivación como formas de un mismo mecanismo de poder entre la constricción y la posibilidad de la acción en el marco de un entramado estructural. La Figura $1^{12}$ ilustra esta teoría.

La socialización es un proceso de naturaleza macrosociológica, pero tiene lugar en el nivel microsociológico, poniendo en relación estructura y acción. En su formulación más original, el individuo internaliza el orden estructural -lo hace suyo-, para reproducirlo mediante la acción. Tanto el funcionalismo estructural (conservador), como las teorías de la reproducción y las de la resistencia (críticas), han incorporado la centralidad de este proceso en su análisis. Sin embargo, otros desarrollos teóricos posteriores han evidenciado que la socialización, en tanto que exenta de los mecanismos del poder, resulta un proceso hermético, parcial e insuficiente para dar cuenta de los procesos microsociológicos que relacionan estructura y acción, focalizando el análisis más en el sujeto y la posibilidad del cambio social.

Así pues, si la sumisión es el mecanismo sociológico de reproducción social, mediante la socialización, y la resistencia termina reproduciendo las estructuras sociales igualmente (Holland y Eisenhart, 1990; McRobbie, 1978; Willis, 1988 y 2003), ha de haber algún otro mecanismo sociológico que nos permita explicar el cambio social en la historia. Ortner (1993) introduce esa posibilidad a través de la acción, pero no repara en el proceso de subjetivación que la literatura revisada vincula con la agencia del sujeto (Butler, 2001; Davies, 2006; Esteban, 2000; Youdell, 2006a, 2006b y 2010).

El sujeto del que se habla en este artículo es el sujeto activo y creativo de la resistencia (Connell, 1987; Dubet y Martuccelli, 1998; Holland y Eisenhart, 1990), siendo la resistencia, entendida como contestación, un espacio de la conciencia que activa la reflexividad (Adkins, 2002 y 2003; Butler, 1997) de la práctica, aun cuando pueda existir constreñimiento sobre esta última. Por lo tanto, el sujeto activo, el de la práctica, es el sujeto agente, el sujeto de la acción social.

12 Esta Figura se encuentra al final del presente artículo (Nota de los editores). 
Para dar cuenta de estos procesos, cuya naturaleza es social, es necesaria una formulación epistemológica mediante dos fenómenos: 1) la acción social, desde una visión postestructuralista; y 2) el devenir sujeto, fenómeno siempre en proceso y consecuente de esa acción social. Así entendido, el sujeto se forma en el despliegue mismo de la acción social. Con ello es posible dar cuenta de la práctica integrando los niveles micro (acción) y macrosociológicos (estructura) de análisis, así como las dimensiones: material (organización material de la estructura social) y simbólica (nivel discursivo) y los procesos sociológicos de reproducción y cambio.

Toda organización estructural tiene dos niveles: macro y mesosociológico. El macro corresponde a la sociedad; el meso, a las instituciones. En ambos casos encontramos una dimensión material, marcada por las condiciones materiales de existencia; y una discursiva o simbólica, formada por valores, ideas, normas, creencias, estereotipos, mitos, etc.: el discurso social dominante y el del entorno institucional inmediato, respectivamente.

En este entramado, el devenir sujeto se activa por dos mecanismos complementarios del poder a través de la acción: estar sujeto a discursos (ver lateral negro de la Figura 1, a la izquierda), y ser sujeto de prácticas (ver lateral gris de la Figura 1, a la derecha). De Butler (1997) se ha tomado la idea de estar y ser sujeto, el resto de la argumentación es mío. Desde una aproximación sociológica, se entiende aquí que la sujeción es a los discursos, pero el ser sujeto sociológico radica en ser sujeto agente o de la acción, el sujeto de las prácticas sociales, las cuales ocurren en el entorno material de cada una de las instituciones, así como de la sociedad en su conjunto, de donde se derivan esos discursos.

De este modo, las prácticas ponen en relación los dos niveles estructurales, el material, que las contextualiza, y el simbólico, que las orienta dentro de ese contexto material. El individuo está sujeto a discursos dominantes. Ahora bien, en tanto que sujeto de prácticas, el sujeto agente puede encauzar sus prácticas en dos direcciones diferentes. Mediante el proceso de socialización, tal como lo plantea la teoría sociológica clásica, el individuo sujeto a esos discursos es sujeto de prácticas de sumisión a los mismos, pues la socialización es el proceso pasivo por el cual el individuo internaliza el orden socioestructural y lo hace subjetivamente suyo, se somete, de alguna forma, a él; de manera que las prácticas resultantes de esa sumisión al discurso dominante son, en última instancia, prácticas de reproducción del orden social establecido, al replegarse al mismo.

Sin embargo, a menudo, el sujeto de la acción ha de hacer frente a discursos contradictorios, procedentes de diferentes instituciones que le rodean y a las cuales pertenece (esto es, con las que tiene una identificación afectiva 
fuerte), como la familia, el grupo de amistad o la pareja, por ejemplo. A veces, las diferencias discursivas se dan incluso dentro de una misma institución. Es esa contradicción entre discursos la que activa la reflexividad de la práctica, poniendo en marcha un proceso de subjetivación simultáneo a la socialización. Las prácticas de resistencia son las formas de acción resultantes de la resolución, al menos puntual, de dichas contradicciones en el sujeto agente, si bien, no se trata de un proceso exento de controversias personales y estructurales, pues, como señala la teoría sociológica, la práctica está constreñida por la estructura.

A pesar de todo, el sujeto agente consigue dar salida, no siempre de manera consciente, a esas contradicciones subjetivas, y desarrollar su acción. La resolución tiene lugar entre la confrontación con el discurso dominante y la ruptura con el mismo. Mientras que la confrontación puede derivar finalmente en sumisión o adhesión, son las prácticas de ruptura con el discurso dominante las que introducen espacios estructurales para un posible cambio respecto al orden social establecido.

Cuando las prácticas introducen cambios en la organización material de una institución, o su sumatoria lo hace sobre una sociedad dada, se van introduciendo cambios, también, en la estructura de lo simbólico; esto es, en el discurso dominante que orienta las prácticas de los sujetos de esa sociedad. De este modo, la interpretación materialista de la acción se dota de elementos postestructuralistas, al incorporar la discursividad en el análisis; y la explicación sobre el devenir sujeto permite dar cuenta, también, de la dinámica de la sociedad.

Esta propuesta procede de una investigación más amplia, sobre política afectivo-sexual (Venegas, 2013a, 2013b), con el objetivo de analizar los valores, normas y prácticas sobre género, sexualidad, afectividad y cuerpo en los regimenes afectivo-sexuales que son barrio, familia, escuela, grupo de amistad y pareja, para estudiar los procesos de socialización y subjetivación que tienen lugar en torno a ello en la adolescencia, como procesos microsociológicos que se corresponden con procesos macrosociológicos de reproducción y cambio, respectivamente, teniendo en cuenta las perspectivas comparativas de clase social, género y etnia.

Para ello, se ha seguido una metodología de investigación acción con un enfoque constructivista. Se ha diseñado e implementado un curso de educación afectivo-sexual con adolescentes de 15-18 años de dos grupos-clase de dos colegios de Granada (España). Tras el curso, se ha realizado una entrevista personal, semiestructurada y en profundidad a cada estudiante de ambos grupos-clase, siendo 42 en total. 
Uno de los ejes centrales del análisis de las entrevistas ha sido, pues, el devenir sujeto. Por razones de espacio, se analiza aquí sólo una de las entrevistas en profundidad, a modo de caso, para ilustrar esta teoría. La entrevista con Raúl, ${ }^{13}$ un chico heterosexual de 17 años, constituye un paseo de dos horas por los vericuetos de su experiencia vital, de la que se analiza aquí la dimensión afectivo-sexual desde el enfoque de la clase, el género y la etnia.

En este caso, el nivel macro corresponde a la sociedad española; el meso, a las instituciones estudiadas en esta investigación: familia, escuela, barrio, grupo de amistad (o de pares) y pareja. En ambos niveles estructurales encontramos una dimensión material -las condiciones materiales de existencia en función de su clase (obrera), su modelo de género (la masculinidad patriarcal) y su etnia (entre la gitana y la castellana)-; y una discursiva o simbólica, formada por los valores, ideas, normas, creencias, estereotipos, mitos, etc. que portan esas tres dimensiones de clase, género y etnia en los discursos tanto dominantes como inmediatos en el entorno institucional al cual pertenece Raúl.

En dicho entramado estructural, la entrevista con Raúl da cuenta de su devenir sujeto mediante dos mecanismos de poder complementarios: estar sujeto a esos discursos dominantes e inmediatos -que constriñen su acción-, y al mismo tiempo ser sujeto de prácticas -lo cual posibilita su acción-. Desde la aproximación sociológica de este trabajo, se observa cómo el discurso que construye Raúl en su entrevista da cuenta del entramado estructural donde está inserto; asimismo, las prácticas que narra dan cuenta de ese espacio simultáneo de constricción y posibilidad que ilustra los procesos de socialización y subjetivación en las cuales se ha definido su devenir sujeto.

Su vida está repleta de vaivenes: la enfermedad de su madre (hasta provocarle la muerte); el abandono de su padre; su decisión, temporal, de vivir con su padre y dejar a su madre sola con su enfermedad. Su socialización primaria transcurre principalmente en el seno de la familia materna extensa, donde los vínculos afectivos son estrechos; la figura matriarcal de la abuela constituye el pilar familiar y uno de sus tíos aparece como su referente de masculinidad. Sin embargo, los valores (conservadores y patriarcales) que posee su familia chocan a menudo con los de su grupo de amistad (más permeado por el cambio social). El resultado es un discurso que habla de una subjetividad marcada por las contradicciones.

Aunque se han separado analíticamente para poder explicarlos, los dos mecanismos (estar sujeto a discursos y ser sujeto de prácticas) ocurren simultáneamente en el discurso del sujeto, si bien, proceden de procesos cronológi-

13 Nombre ficticio para mantener su anonimato. 
camente vividos en su devenir sujeto, pues la socialización primaria ocurre en la infancia, mientras que la subjetivación es más propia de la adolescencia. A continuación se analizan algunos fragmentos del discurso de Raúl, para ilustrar todo ello.

El barrio donde se ha dado la socialización de Raúl constituye un universo generizado, que es el marco de referencia de su subjetivación. Así, ha internalizado una división sexual del trabajo que define un modelo de clase, género y etnia incuestionable para él:

Una mujer digna es la que trabaja en su casa y no trabaja fuera. Es como si una mujer digna, es como un escalón más, ¿̇me entiendes? Mi abuelo trabajaba, y ganaba, pues mi abuela nunca la ha dejao trabajar mi abuelo. Nunca la ha dejao trabajar en ná. Pues eso significa que mi abuelo ha tenío dos cojones pa mantener a mi abuela y a tó la familia. Pues eso se nota en las casas. Mira, una mujer que trabaja no es que sea más digna o menos digna, pero yo qué sé, tiene menos prestigio o como se diga eso.

La homofobia presente en su entorno es otro elemento central en su devenir sujeto afectivo-sexual:

"Mi abuelo no soporta a los mariquitas. Es cerrao. Dice que después de tener un niño drogao, tener un niño maricón es... eso. Mi tío tampoco los soporta, como es mu, mu, mu macho. Le da asco".

En ese universo, los hombres son rudos y las mujeres invierten en capital corporal, porque ello revierte en capital simbólico, social y económico en el "espacio de la subasta sexual" (Holland y Eisenhart, 1990).

Raúl se construye como sujeto masculino en la contradicción entre una masculinidad "hegemónica" (Connell, 1987), "viril" (Bourdieu, 2005), que constriñe la acción obligando a rechazar en público aquellas prácticas consideradas femeninas, al tiempo que ha sido socializado en su aprendizaje:

Me dice "haz tu cuarto". Pos yo voy con el trapo, como a mí me han enseñao de tó la vida, pos yo voy con mi trapo, que no se entere nadie, que me da vergüenza, le digo a mi abuela que a nadie se lo cuente.

En tanto, el grupo de amistad es el espacio de formación del sujeto afectivo-sexual adolescente por excelencia (McRobbie, 1978; Holland y Eisenhart, 1990; Willis, 1988). En él se construyen los procesos sociales de liderazgo en función de la experiencia sexual, imponiéndose una "virilidad como nobleza” (Bourdieu, 2005), que obliga, una vez más, a dar muestras públicas de ella. Así, Raúl reconoce que "cuanto más ligues, es mejor, más macho eres, tú me entiendes, ¿no? O... te lo tienes hasta que inventar pa que no quees en ridículo. Me está dando vergüenza”.

Algunos elementos emergentes en su entorno contienen, sin embargo, un potencial de cambio social intergeneracional que le ofrecen referentes 
para resistir (contestar) a los discursos dominantes que han definido su socialización. Así, reconoce que "las niñas de la clase, una va a ser maestra, la otra va a ser de tiendas..."; por lo tanto, la siguiente generación de mujeres de su entorno no tiene que quedarse ya en casa para ser digna, como tuvo que hacerlo la generación de su abuela.

Este indicio de cambio es fundamental en ese contexto de clase obrera y con un volumen importante de población gitana, y está relacionado con cambios estructurales más macrosociales, derivados del acceso de las mujeres a educación formal, mercado laboral y movilidad social desde la segunda mitad del siglo XX (Beck y Beck-Gernsheim, 2001; Bourdieu, 2005; Giddens, 2004; Venegas y Lozano, 2016).

Pero es su relación afectivo-sexual con una chica de la clase la experiencia que mayores conflictos le genera:

Me arrepiento de lo que hice [en el viaje de estudios]. No te voy a contar, porque me da vergüenza. Ella me puso la mano en las partes prohibidas de las niñas [...] ¿Cómo te lo digo? Una teta. Entonces, yo digo "si me ha dejao que le toque esto, me dejará que le toque otras cosas". Pos hubo tocamiento, y ya está. Luego hubo lo que hubo. Yo qué sé. Porque yo me ofrecía y ella se ofrecía, pos amos, ¿no? Nos dejaron solos, pero que el mal rato se pasa, ¿eh? Mi primera vez fue... [...] No puedo parar de pensar en eso, no sé qué me pasa. No puedo estudiar, me pongo a, no me lo quito de la cabeza. [...] Yo creo que la primera vez de ella no ha sío, ¿eh? Porque no le salía sangre ni ná de eso [...] Luego me sentí sucio. [...] Es como si yo sintiera que no lo tenía que haber hecho, porque no la quiero. No... no, yo sé que mi vida no la voy a compartir con ella [...] No fue como las parejas, que se ponen... [...] No era lo que yo creía. [...] Yo nunca hubiera abusao de ella, yo con eso soy muy cuidadoso [...] Es sucio y no hice apenas ná. [...] Yo placer no sentí, yo sentí que, que eyaculé, ya está. Pero ella tampoco tuvo que sentir ná, ¿no? porque se echa un agua, ¿no? [...] Pero es que, cuando estoy con ella, me siento bien y me gusta, ¿qué es lo que me pasa? [...] No la quiero, a lo mejor, la dignidad se la he quitao yo y yo no le correspondo, ¿̇tú me entiendes? Por eso me siento sucio.

Raúl ha internalizado un modelo de relación afectivo-sexual sustentado en la posesión y los celos, legitimados patriarcalmente de manera implícita, y donde se impone un doble estándar de género: "Si yo le doy un abrazo a una niña, ellas son como mis hermanas. [...] Ella sabe que me da coraje. Que no es lo mismo una niña que un niño. [...] Y yo noto que no me quiere, porque si me quisiera de verdad, ya vería lo que hace".

Como se ve a lo largo de todo el discurso, la vergüenza es el vector que da cuenta de los conflictos y contradicciones a los que ha de hacer frente Raúl en su devenir sujeto, entre las imposiciones normativas donde se ha socializado como actor social y las referencias alternativas que sopesa en su búsqueda de autonomía como sujeto agente. 
Este fragmento de la entrevista muestra la colisión continua, en el devenir sujeto afectivo-sexual adolescente, entre los discursos dominantes (el social sobre la masculinidad viril; el familiar sobre la honra; el del grupo de amistad sobre la promiscuidad; el adolescente sobre el inicio de la experiencia sexual) y las prácticas concretas, los sentimientos, los miedos, los tabúes, lo estereotipos, los prejuicios, las falsas creencias.

El suyo es un ir y venir entre la sujeción a los discursos que le rodean y las prácticas de contestación a esos discursos, entre la necesidad de pertenencia a los grupos de los cuales forma parte y la búsqueda de su individualidad. La contestación a esos discursos activa la reflexividad de su práctica, que oscila entre la sumisión y la contestación, más próxima a la confrontación adolescente que a la ruptura con los modelos dominantes que definen su entorno social.

Así pues, el lugar que él ocupa subjetivamente en ese universo de clase, género y etnia presenta elementos contradictorios, los cuales constituyen las piezas que ha de encajar en su devenir sujeto, entre una socialización bajo un modelo patriarcal, machista y homófobo, y una subjetivación que le lleva a distanciarse de ese modelo, buscando su autonomía personal a través de formas más igualitarias de ser sujeto afectivo-sexual, que van incorporando al contexto estudiado en la investigación algunos agentes como las familias más igualitarias del barrio, la escuela, o las chicas y chicos que no se adhieren a ese modelo patriarcal. Todo ello es objeto de la investigación; si bien excede en este artículo por razones de espacio.

\section{Conclusiones}

El presente trabajo ha emprendido un recorrido cronológico por la teoría sociológica, desde un enfoque feminista, reparando en, y debatiendo, algunos hitos teóricos de relevancia para la compresión del devenir sujeto, entre la socialización y la subjetivación, la constricción y la posibilidad. Con ello se ha buscado tanto recoger las principales aportaciones al respecto, como discutir sus debilidades a fin de superarlas mediante la formulación de una propuesta sintetizadora y holística del devenir sujeto, a través de una lectura postestructuralista de la teoría de la acción.

Esta teoría se refiere al devenir sujeto, fruto de un mecanismo de poder que se despliega mediante un proceso doble y simultáneo: estar sometido a y ser sujeto de prácticas. Con estas últimas el sujeto deviene agente, esto es, el sujeto de la acción. Una acción que se debate entre la constricción y la posibilidad en tanto que la acción estructuralmente situada es una acción relacional y, por ende, mediada por el poder, que es tanto externo al sujeto como su propia jurisdicción. 
De este modo, acción, sujeto y estructura quedan interconectadas en procesos de mantenimiento de las estructuras sociales, a través de la socialización, y la consecuente sumisión, que conduce al sujeto a adherirse a ese orden estructural; pero también de desafío a esas estructuras sociales, mediante la subjetivación que hace posible la resistencia, entendida como contestación, que activa, a través de la reflexividad de la práctica, la posibilidad de cambio social.

Para ilustrar todos estos procesos, el artículo ha mostrado el devenir sujeto afectivo-sexual de un adolescente de 17 años, heterosexual, que vive en un barrio de clase obrera de la ciudad de Granada (España) y cuya familia pertenece, en parte, a la etnia gitana. El análisis muestra las dinámicas de un sujeto que oscila en un universo de clase, género y etnia caracterizado por el conflicto y la contradicción entre una socialización bajo un modelo patriarcal, machista y homófobo, y una subjetivación que busca distanciarse de ese modelo, para construir su autonomía personal mediante un modelo más igualitario de ser sujeto afectivo-sexual.

\section{Referencias}

Adkins, Lisa (2002), Revisions: gender and sexuality in late modernity, Reino Unido: Open University Press.

Adkins, Lisa (2003), "Reflexivity. Freedom or Habit of Gender?", en Theory, Culture and Society, año 20, Reino Unido: Sage.

Allen, Louisa (2005), Sexual Subjects. Young people, sexuality and education, Estados Unidos: Palgrave McMillan.

Barret, Michèle (1992), "Words and Things: Materialism and Method in Contemporary Feminist Analysis", en Barret, Michèle y Phillips, Anne [eds.], Destabilizing Theory: Contemporary Feminist Debates, Reino Unido: Polity.

Bartky, Sandra Lee (1988), "Foucault, Femininity, and the Modernization of Patriarchal Power", en Diamond, Irene y Quinby, Lee [eds.], Feminism and Foucault. Reflections on Resistance, Estados Unidos: Northeastern University Press.

Beck, Ulrich y Beck-Gernsheim, Elisabeth (2001), El normal caos del amor, España: Paidós. Berger, Peter y Luckmann, Thomas (2008), La construcción social de la realidad, Argentina: Amorrortu.

Bordo, Susan (1988), "Anorexia Nervosa: Psychopathology as the Crystallization of Culture", en Diamond, Irene y Quinby, Lee [eds.], Feminism and Foucault. Reflections on Resistance, Estados Unidos: Northeastern University Press.

Bordo, Susan (1995), Unbearable Weight. Feminism, Western Culture and the Body, Estados Unidos: University of California Press.

Bourdieu, Pierre (2005), La dominación masculina, España: Anagrama.

Butler, Judith (1993), Bodies that matter. On the discursive limits of sex, Reino Unido: Routledge. 
Butler, Judith (1997), The psychic life of power. Theories in subjection, Estados Unidos: Stanford University Press.

Butler, Judith (2001), "La cuestión de la transformación social", en Beck-Gernsheim, Elisabeth et al., Mujeres y transformaciones sociales, España: El Roure.

Cervulle, Maxime et al. [coords.] (2012), "Subjectivités et rapports sociaux" en Cahiers du Genre, núm. 53, Francia: L’ Harmattan.

Connell, Robert William (1987), Gender and Power. Society, the Person and Sexual Politics, Estados Unidos: Stanford University Press.

Connell, Robert William (2002), Gender, Reino Unido: Polito Press.

David, Miriam et al. [coord.] (2006), “Troubling identities: reflections on Judith Butler's philosophy for the sociology of education", en British Journal of Sociology of Education, año 27, Reino Unido: Taylor and Francis.

Davies, Bronwyn (2006), "Subjetification: the relevance of Butler's analysis for education", en British Journal of Sociology of Education, año 27, Reino Unido: Taylor and Francis.

Dubet, Françios y Martuccelli, Danilo (1996), “Théories de la socialisation et définitions sociologiques de l'école", en Revue française de sociologie, año 37, Francia: Presses de Sciences Po.

Dubet, François y Martuccelli, Danilo (1998a), Dans quelle société vivons-nous?, Francia: Édition du Seuil.

Dubet, François y Martuccelli, Danilo (1998b), En la escuela. Sociología de la experiencia escolar, Argentina: Losada.

Durkheim, Ėmile (1975), Educación y sociología, España: Península.

Esteban, Mari Luz (2000), "Promoción social y exhibición del cuerpo", en Del Valle, Teresa [ed.], Perspectivas feministas desde la antropología social, España: Ariel.

Esteban, Mari Luz (2001), "El género como categoría analítica. Revisiones y aplicaciones a la salud”, en Miqueo, Consuelo [ed.], Perspectivas de género en salud. Fundamentos cientificos y socio-profesionales de diferencias sexuales no previstas, España: Minerva Ediciones.

Foucault, Michel (1989), Historia de la sexualidad vol. I. La voluntad de saber, Madrid: Siglo XXI.

Giddens, Anthony (2004), La transformación de la intimidad, Madrid: Cátedra.

Gil, Silvia (2013), "Feminismos contemporáneos en la crisis del sujeto. Hacia una política de lo común”, en Díaz, Capitolina y Dema, Sandra, Sociología y género, España: Biblioteca Universitaria de Editorial Tecnos.

Grosz, Elisabeth (1987), "Notes towards a corporeal feminism", en Australian Feminist Studies, año 5, Australia: Routledge.

Holland, Dorothy y Eisenhart, Margaret (1990), Educated in Romance. Women, Achievement, and Collage Culture, Estados Unidos: The University of Chicago Press.

Maquieira, Virginia (2001), "Género, diferencia y desigualdad", en Beltrán, Cristina y Maquieira, Virginia, Feminismos, España: Alianza.

McRobbie, Angela (1978), "Working Class Girls and the Culture of Femininity", en Women's Studies Group, Women take issue, Reino Unido: Hutchinson.

McRobbie, Angela (1994), Postmodernism and Popular Culture, Reino Unido: Routledge.

Narotzky, Susan (1995), Mujer, mujeres, género. Una aproximación crítica al estudio de las mujeres en las ciencias sociales, España: CSIC.

Nicholson, Linda J. [ed.] (1990), Feminism/Postmodernism, Reino Unido: Routledge. 
Ortner, Sherry (1993), "La teoría antropológica desde los años sesenta”, en Cuadernos de Antropología, México: Universidad de Guadalajara.

Quintana, Laura y Manrique, Carlos [coords.] (2012), "Las técnicas de poder y formas de vida: otras perspectivas en torno a la biopolítica”, en Revista de Estudios Sociales, núm. 43, Colombia: Universidad de los Andes.

Skeggs, Beverly (1997), Formations of Class and Gender. Becoming respectable, Reino Unido: Sage.

Touraine, Alain (2005), Un nuevo paradigma para comprender el mundo de hoy, España: Paidós.

Venegas, Mar (2012), “Socialización y subjetivación. El caso de las relaciones afectivosexuales adolescentes”, en Venegas, Mar [ed.], La sociología y los retos de la educación en la España actual, España: Germanía.

Venegas, Mar (2013a), Amor, sexualidad y adolescencia. Sociología de las relaciones afectivosexuales, España: Comares.

Venegas, Mar (2013b), "Sex and relationships education and gender equality: recent experiences from Andalusia (Spain)", en Sex Education: Sexuality, Society and Learning, año 13, Reino Unido: Routledge.

Venegas, Mar y Lozano, Antonio (2016), "Cambio social y cambio en los modelos de familia: trabajar por la igualdad”, en Becerril, Diego y Venegas, Mar [eds.], La custodia compartida en España, España: Dykinson.

Weeks, Jeffrey (2003), Sexuality, Reino Unido: Routledge.

Weiss, Eduardo (2012), “Los estudiantes como jóvenes. El proceso de subjetivación”, en Perfiles Educativos, año 34, México: Universidad Nacional Autónoma de México.

Willis, Paul (1988), Aprendiendo a trabajar, España: Akal.

Willis, Paul (2003), "Foot Soldiers of Modernity: The Dialectics of Cultural Consumption and the 21st-Century School", en Harvard Educational Review, año 73, Estados Unidos: Harvard University, Graduate School of Education.

Youdell, Deborah (2006a), "Diversity, Inequality, and a Post-structural Politics for Education”, en Discourse: Studies in the Cultural Politics of Education, año 27, Reino Unido: Taylor and Francis.

Youdell, Deborah (2006b), "Subjectivation and performative politics - Butler thinking Althusser and Foucault: intelligibility, agency and the racednationedreligioned subjects of education", en British Journal of Sociology of Education, año 27, Reino Unido: Routledge.

Youdell, Deborah (2010), "Recognizing the subjects of education", en Apple, Michael et al. [eds.], The Routledge International Handbook of the Sociology of Education, Reino Unido: Routledge. 


\section{Anexo}

\section{Figura 1}

\section{Devenir sujeto}

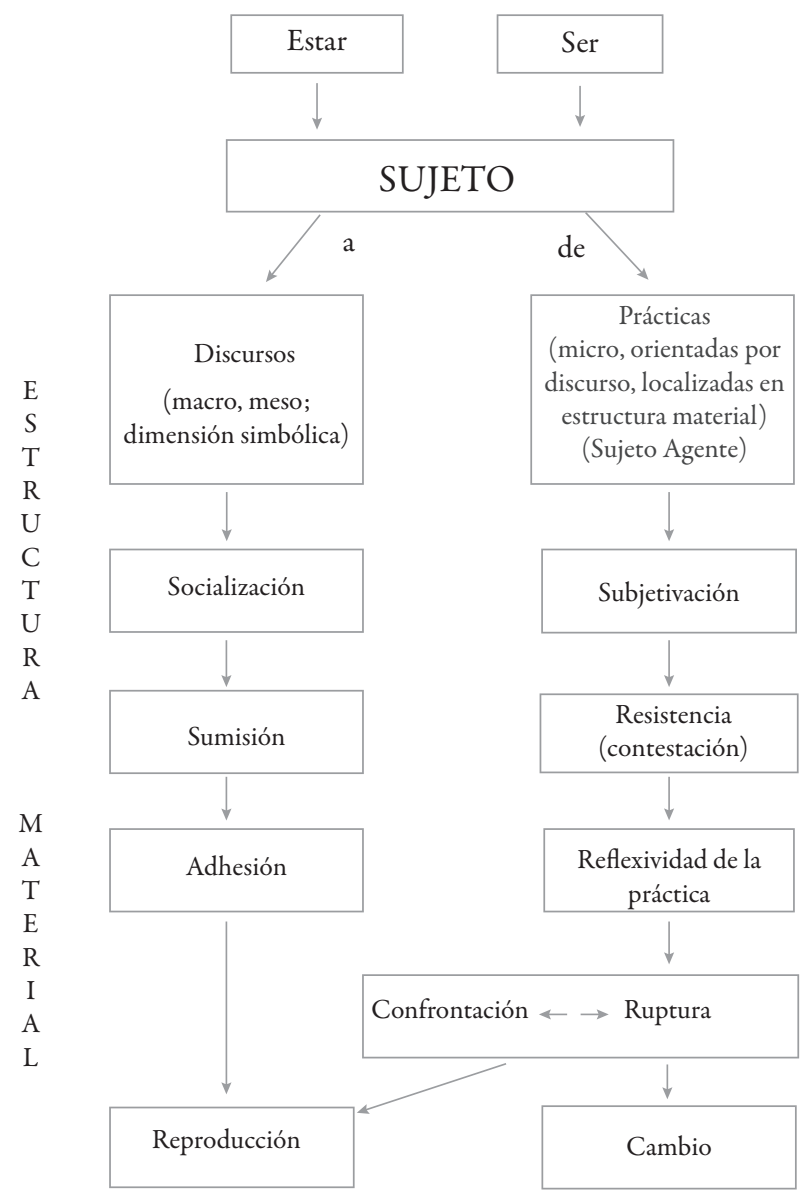

Fuente: Venegas (2013a). 
Mar Venegas. Profesora del Departamento de Sociología, Universidad de Granada, España. Doctora en Sociología y Experta en Género e Igualdad de Oportunidades por esta Universidad. Líneas de investigación: estudios de género y sexualidad desde la sociología de la educación, y la sociología del profesorado. Publicaciones recientes: Venegas, Mar, "El cuerpo en la escuela de las ausencias. La educación afectivosexual en la batalla entre coeducar o segregar", en Revista Iberoamericana de Educación / Revista Ibero-americana de Educação, vol. 64/1, Madrid y Buenos Aires: Organización de Estados Iberoamericanos para la Educación, la Ciencia y la Cultura (OEI), Centro de Altos Estudios Universitarios (CAEU) (2014); Venegas, Mar, "Investigar las relaciones afectivosexuales: el diseño de un cuestionario abierto", en Empiria. Revista de Metodología en Ciencias Sociales, vol. 28, Madrid: Universidad Nacional de Educación a Distancia (UNED) (2014); Venegas, Mar, "Segregar versus coeducar: Un marco para educar las relaciones afectivosexuales y prevenir la violencia de género en la adolescencia”, en Ehquidad. International Welfare Policies and Social Work Journal, núm. 3, Logroño: Asociación Internacional de Ciencias Sociales y Trabajo Social (2015).

Recepción: 12 de octubre de 2015.

Aceptación: 9 de agosto de 2016. 